\title{
The Multi-scale Model Study on Steel Latticed Arch
}

\author{
Liu Mengsha ${ }^{1, a}$, Li Jingyuan ${ }^{1, b}$, Ju Jinsan ${ }^{1,2, c}$
}

The Key Laboratory of Agricultural Facility, Department of Agriculture, Beijing, China

Department of Civil Engineering, China Agricultural University, Beijing, China

aliumengsha@cau.edu.cn, 'blijingyuan@cau.edu.cn, cjujinsan@cau.edu.cn

Keywords: Multi-scale model, Element beam model, Steel latticed arch, Numerical simulation

\begin{abstract}
In this study, Ansys was used to build a detailed finite element model of the steel latticed arch. The multi-scale model was adopted to analyze the behavior of the steel latticed arch and the finite element analysis results were analyzed comparing with the element beam model. The conclusion can be obtained as follows: The stress distribution of multi-scale model is basically the same with the beam element mode, but the stress and vertical displacement of the force point showed a great difference between the two kinds of models; the ultimate bearing capacity of the multi-scale model is about $40 \%$ of the beam element model.
\end{abstract}

\section{Introduction}

In numerical simulation, the differences of calculation results caused by different element types are obvious. Although the macroscopic model has the advantage in simple model building and small computational amount, the local buckling failure can't be obtained through the calculation. And the microcosmic failure mechanics can be reflected by microcosmic model set up by the solid elements, but the Computer computing ability and the complexity of modeling limit its actual use. The emergence of the multi-scale model can effectively solve this problem. In the multi-scale model, the detailed model was set up in the local parts that need a detailed analysis and the other parts use macroscopic model to simulate. It can be better grasp that the whole stress characteristics of the structure and the micro failure process.

Many scholars have studied the mechanical properties of steel latticed arch by numerical simulation, but the research based on the multi-scale model is relatively rare. Because of the large model in size and the complex node model, the computing process will take a large amount of time if the overall detail model is created. So the multi-scale model used in the calculation of the steel latticed arch is necessary.

In this study, the multi-scale model was used to research mechanical behavior of the steel latticed arch. The model was set up according to the concrete stress distribution of the structure. And the comparison between the multi-scale model and the beam element model was carried out to verify the feasibility of the multi-scale model in the study on steel latticed arch.

\section{FINITE ELEMENT MODEL}

\subsection{Model geometry information}

This study adopted the steel latticed arch as the research object. The geometry information is indicated by Fig. 1 . The arch is $10 \mathrm{~m}$ in span length and $1.5 \mathrm{~m}$ in height.

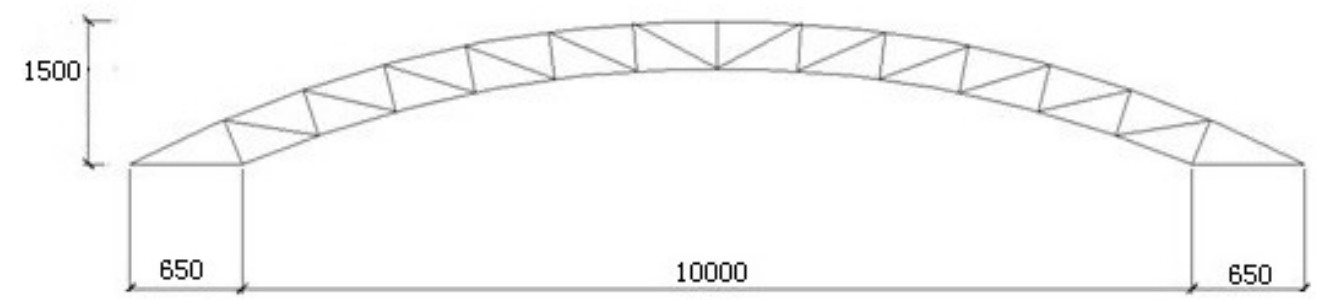

Fig. 1 The geometry information of the steel latticed arch 
The cross section of the arch is a triangle whose height is $0.5 \mathrm{~m}$ and base is $0.6 \mathrm{~m}$. Square tubes which are $0.04 \mathrm{~m}$ in side length and $0.001 \mathrm{~m}$ in thickness were used in the chord members and the web members. The constitutive relation model adopted in the numerical simulations is linear hardening model. The elasticity modulus $=2.06 \times 1011$, hardening modulus $=6.21 \times 109$ and the Poisson ration $=0.3$.

\subsection{The beam element model}

The beam element model is presented in Fig. 2. Three-dimensional linear interpolation beam units were adopted in the element beam model.

\subsection{The multi-scale model}

The multi-scale model is shown in Fig.3. The detail model was set in the loading point and the other position is still the beam element model. The detail part of the model of the loading point is illustrated in Fig.4. In this multi-scale model, the beam elements were set up by the three-dimensional linear interpolation beam units and the shell elements four nodes reduced integral units were used to simulate the detail part of the model.

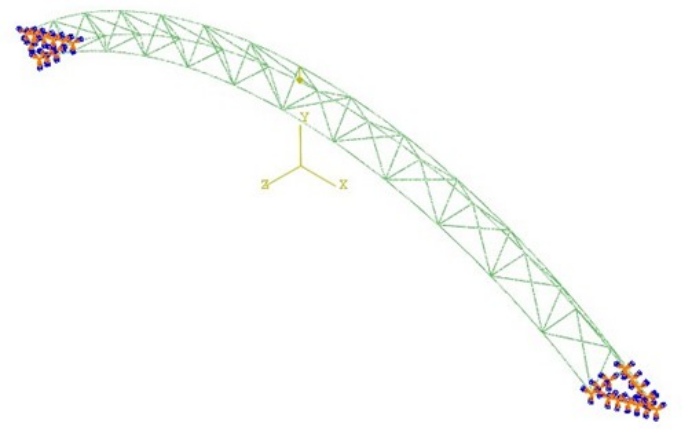

Fig.2 The beam element finite element model of the steel latticed arch

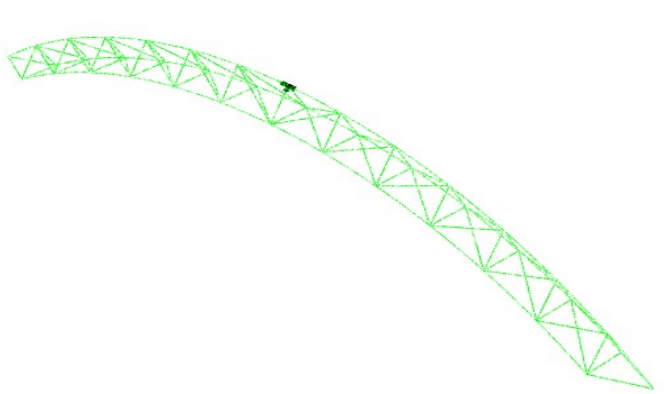

Fig.3 The multi-scale model of the steel latticed arch

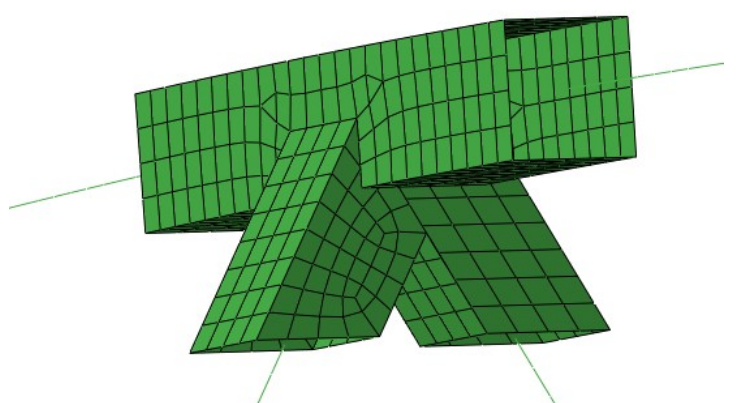

Fig.4 The detail part of the model

\section{The inner force analysis}

The boundary constraint and the loading conditions are the same in the beam element model and the multi-scale model. Supports of the steel latticed arch were constrained on the translation and the rotational degrees of freedom. A $100 \mathrm{~N}$ vertical downward force was applied on the arch crown and the load time was $1 \mathrm{~s}$.

The inner force analysis of the steel latticed arch was conducted through the numerical simulation. The axial force diagrams are shown in Fig. 5 as the load time is equal to 1. By contrast, both the position and value of the axial pressure and the axial tensile, and the distribution of internal forces in the multi-scale model coincides with the beam element model. The maximal axial pressure area is located in the upper chord members closed to the arch crown and the maximal axial tensile area is located in the two web members closed to the load point. 


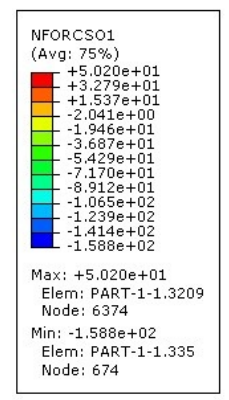

a. The axial force diagrams of the beam element model
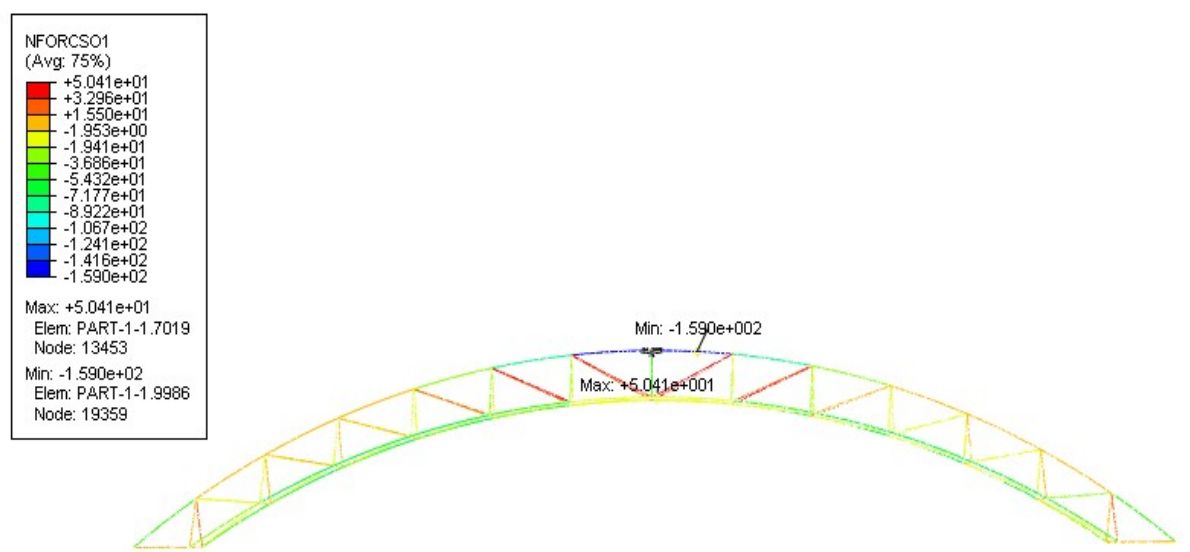

b. The axial force diagrams of the multi-scale model

Fig.5 The axial force diagrams of the steel latticed arch

Fig.6 presents the Von Mises Stress of the entire structure. The maximum stress of multi-scale model is $6.702 \mathrm{x} 107 \mathrm{~Pa}$, is greater than the maximum stress in the beam element model. The maximum stress appears in the location of the load point in the multi-scale model and the beam element model. The larger stress of the multi-scale model in the load point is caused by the stress concentration phenomenon leading to considerable local stress. And getting rid of the stress concentration, the stress distribution in the multi-scale model is consistent with the beam element model. The multi-scale model can well response the detailed stress distribution and this reflects the advantage of the multi-scale model relative to the macroscopic model.

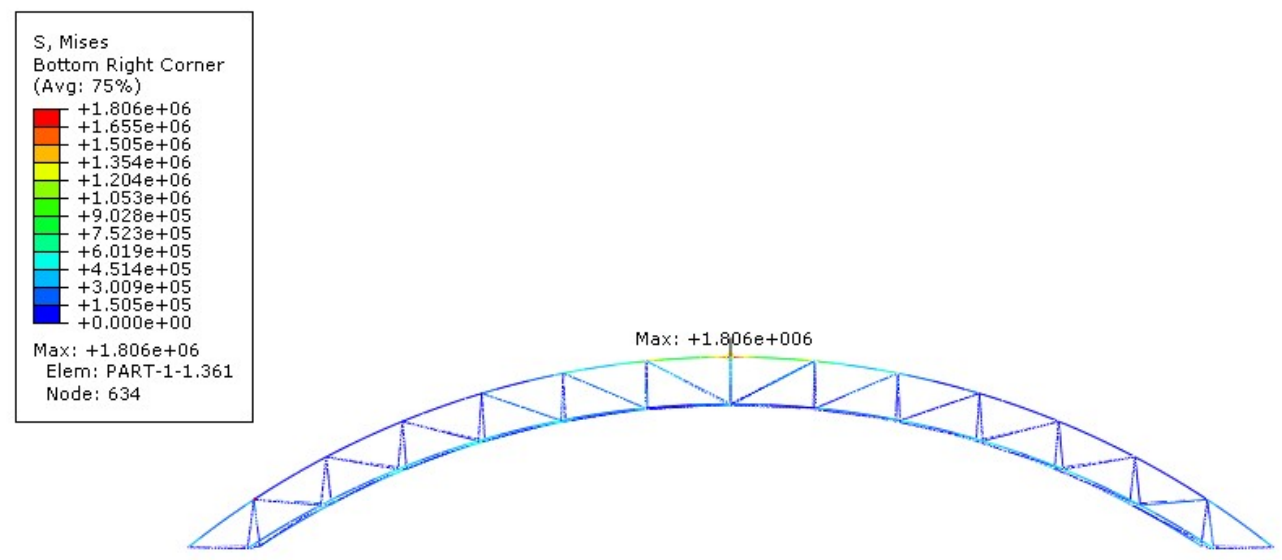

a. The Von Mises Stress of the beam element model 


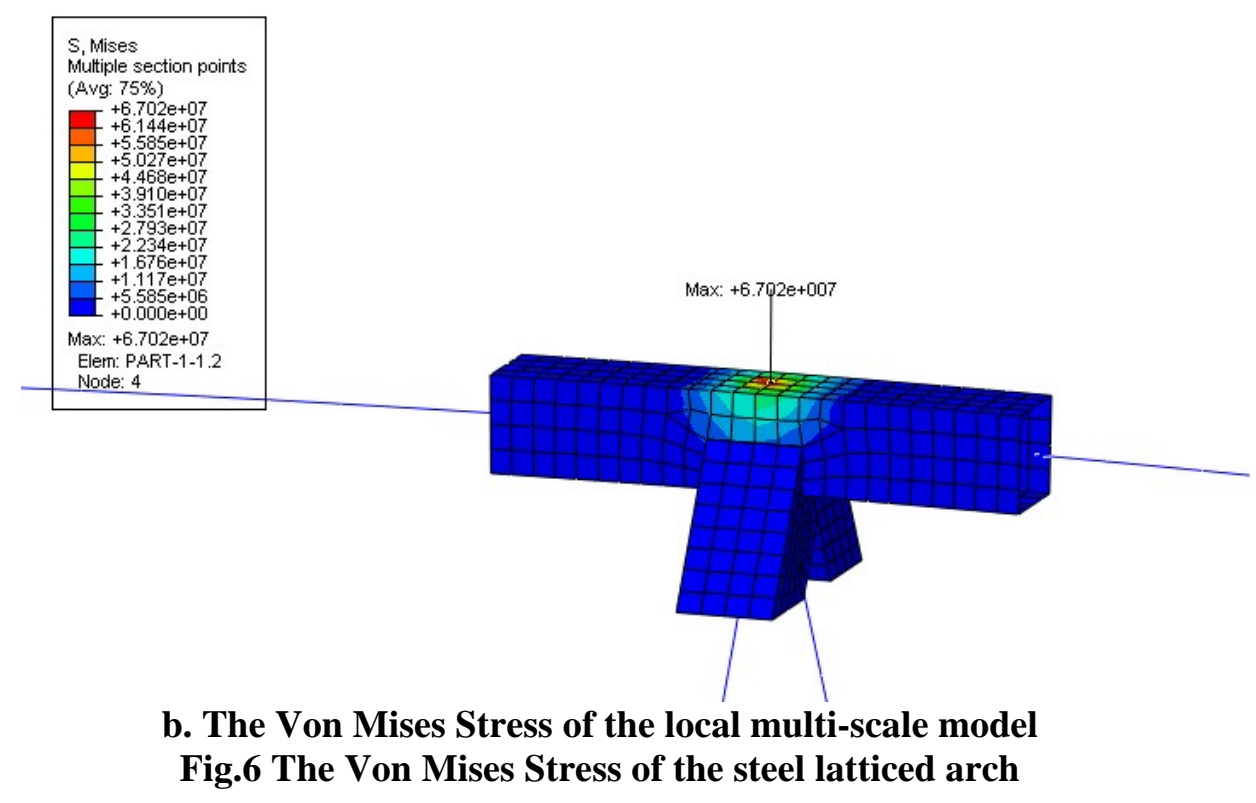

\section{The ultimate bearing analysis}

In this part, the material and geometrical nonlinearities were taken into account to carry out the ultimate bearing analysis of the steel latticed arch.

Fig.7 shows the first buckling mode of the steel latticed arch model under the action of concentrated force applying in the arch crown. From the first buckling mode of the beam element model, it can be observed that the buckling of the upper chord members closed to the load point lead to the structural buckling. And from the first buckling mode of the multi-scale model, it can be observed that the severe deformation in the load point leads to the structural buckling. The first buckling mode was taken as the distribution mode of the initial geometrical imperfection. The initial imperfection, 1/1000 of the span, was taken to carry out the ultimate bearing analysis.

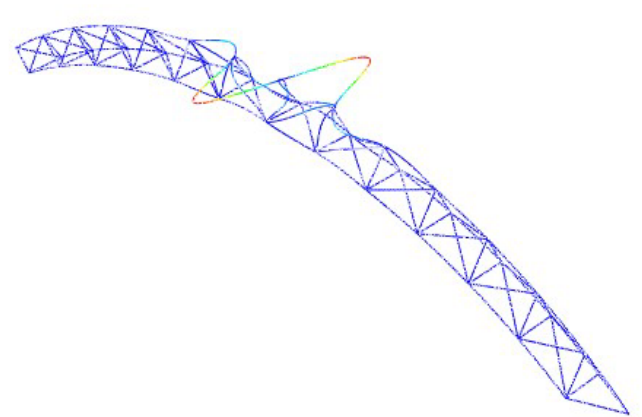

a. The first buckling mode of the beam element model

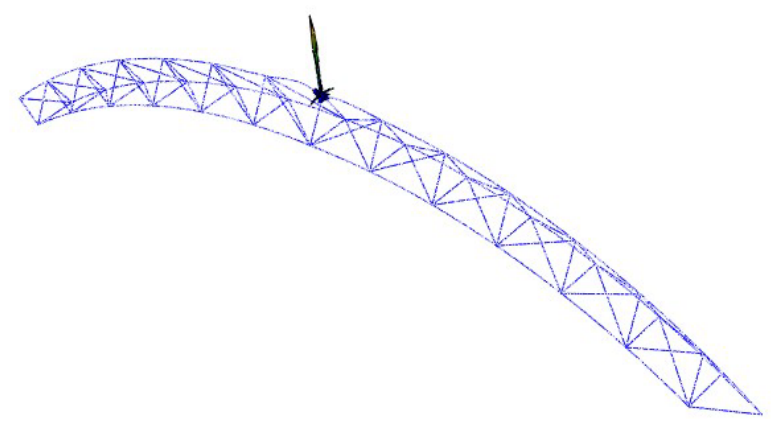

b. The first buckling mode of the multi-scale model

Fig.7 The first buckling mode of the steel latticed arch

The arc-length method was taken to realize the nonlinear analysis and the material and geometric nonlinear were taken into consideration to carry out the ultimate bearing analysis. The concentration load of $50000 \mathrm{~N}$ was applied on the arch crown. The load-displacement curves were got through numerical simulation shown in Fig.8. From the Fig.8, it can be observed that the ultimate bearing capacity of the beam element model is $31000 \mathrm{~N}$ and the ultimate bearing capacity of the multi-scale model is $12600 \mathrm{~N}$. 


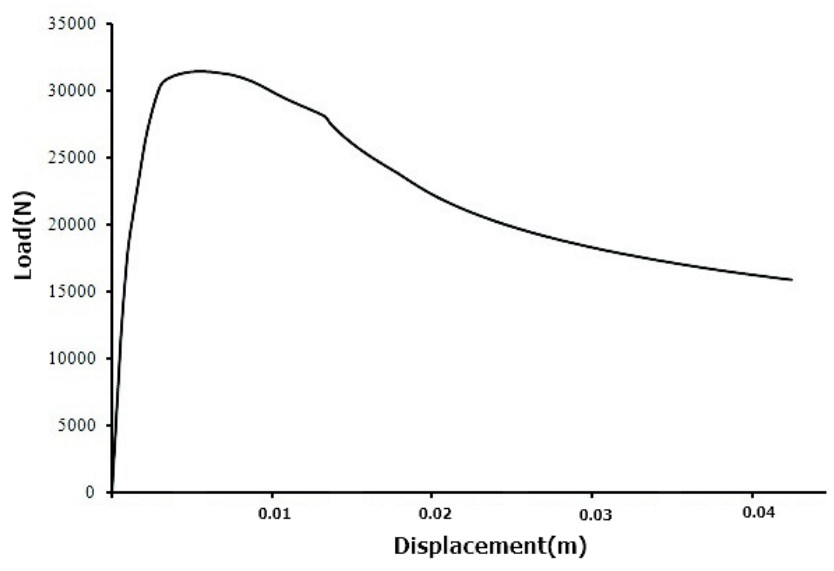

a. The load-displacement curve of the beam element model

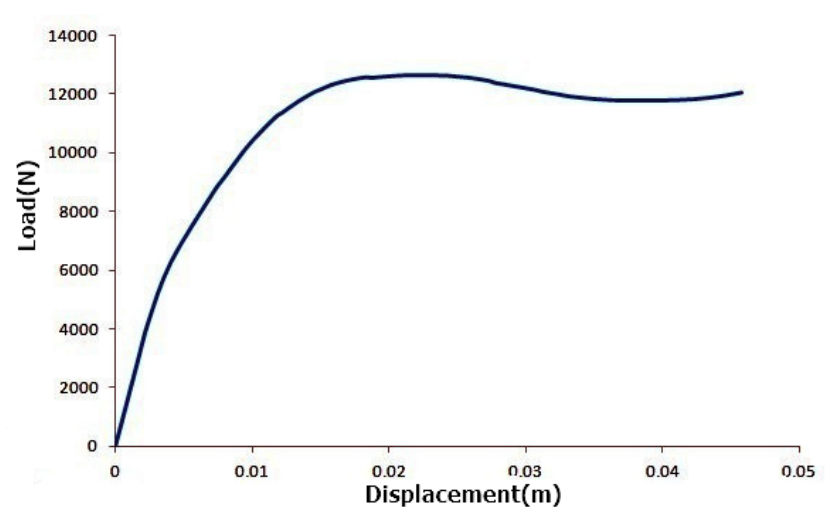

b. The load-displacement
curve of the multi-scale model

Fig.8 The load-displacement curves of the steel latticed arch

The ultimate bearing capacity of the multi-scale model is about $40 \%$ of the beam element model. From the observing of the load-displacement curve of the beam element model, the bearing capacity dropped significantly with the load reaching $31000 \mathrm{~N}$. But in the multi-scale model, the bearing capacity can remain from dropping when the load reached the ultimate bearing capacity. The shapes of curves show that the buckling of global structure lead to the beam element model failure and the multi-scale model failure is caused by the local buckling of the load point. So the multi-scale model can still bear the load after local buckling while the beam element model loses bearing capacity when the load reached the ultimate bearing capacity.

\section{CONCLUSIONS}

In this research, the multi-scale model was adopted to analyze the behavior of the steel latticed arch and the results were analyzed comparing with the element beam model. The first buckling mode was taken as the distribution mode of the initial geometrical imperfection to carry out the ultimate bearing capacity. The analytical results can be obtained from finite element numerical simulation as follows:

(1) The stress distribution of the Multi-scale model is basically the same with the beam element model removing the detail structure located in the load point. But in the load point, the stress in the Multi-scale model is larger than in the beam element model. The larger stress reflects the stress concentration and it conforms to the actual stress distribution.

(2) The ultimate bearing capacity calculated by the beam element model is about twice as much as the capacity calculated by the multi-scale model. With the analysis of the load-displacement from simulation calculation, it could be assumed that the buckling of global structure lead to the beam element model failure and the multi-scale model failure is caused by the local buckling of the load point. So the ultimate bearing capacity calculated by the multi-scale model is less and this simulation can give a safe estimation.

\section{Acknowledgements}

This work was financially supported by National Science Foundation of China (51279206) and The Key Laboratory of Agricultural Facility’s Open Projects (201504).

\section{References}

[1]. Khandelwal K. Multi-Scale Computational Simulation of Progressive Collapse of Steel Frames.[J]. Dissertations \& Theses - Gradworks, 2008. 
[2]. Ladeveze P, Nouy A, Loiseau O. A multiscale computational approach for contact problems[J]. Computer Methods in Applied Mechanics \& Engineering, 2002, 191(43):4869-4891.

[3]. Y. J. Shi, M. Wang, Y. Q. Wang. The seismic performance analysis of steel framework based on the multi-scale model[J]. Engineering Mechanics, 2011, 28(12):20-26.

[4]. Y. L. Zhang, X. M. Guo. The research progresses of the multi-scale simulation and calculation[J]. Chinese Journal of Computational Mechanics, 2011, 28(B04):1-5.

[5]. C. Y. Wang. The analytical method of the multi-scale model[J]. Complex Systems and Complexity Science, 2004, 1(1):9-19.

[6]. Z. H. Sun, Z. X. Li, H. T. Chen. The experimental study on the structure multi-scale finite element model[J]. Special Structure, 2008, 25(4). 